\title{
Mass preparation and technological development of an antifreeze protein
}

\author{
- Toward the practical use of biomolecules -
}

\author{
Yoshiyuki Nishimiya, Yasuhiro Mie, Yu Hirano, Hidemasa Kondo, Ai Miura and Sakae Tsuda*
}

[Translation from Synthesiology, Vol.1, No.1, p.7-14 (2008)]

\begin{abstract}
Antifreeze protein isolated from the blood serum of Arctic and Antarctic fish is an extraordinary biomolecule that binds to ice and preserves cell structure. We recently discovered that Japanese edible fish species also contain an antifreeze protein, and established a method of isolating the protein from fish muscles. We determined that the isolated antifreeze protein consists of a mixture of many isoforms that together are more active than any single isoform. Mass preparation of antifreeze protein is currently under investigation to provide material for a variety of studies and industries.
\end{abstract}

Keywords: Antifreeze protein, 3D structure, ice-binding, mass preparation, cell preservation, ice nucleus plate

\section{Objective}

Antifreeze protein (AFP) can inhibit ice-growth by accumulating on the surface of ice nuclei formed in nearfreezing water, and can preserve cell function under hypothermic conditions $\left(-0{ }^{\circ} \mathrm{C}\right)$. The objective of this study was to develop new technologies so these properties of AFP can be applied in industry and medicine. Figure 1 compares the principles of current cryotechnology without AFP and the expected cryotechnology utilizing AFP. Common perception is that water freezes at $0{ }^{\circ} \mathrm{C}$; however, spontaneous freezing at $0{ }^{\circ} \mathrm{C}$ hardly occurs when liquid water is placed in cold environment such as in the refrigerator $\left(-18^{\circ} \mathrm{C}\right)$. Unfrozen water below $0{ }^{\circ} \mathrm{C}$ is generally called supercooled water ${ }^{[1]}$. Freezing is triggered by natural formation of numerous ice nuclei in supercooled water (Figure 1A, top). The generated ice nuclei successively undergo crystal growth by adsorption of surrounding water molecules, and finally occupy the whole space (Figure 1A, bottom). Thus, a common block of ice is inevitably polycrystalline and not a single crystal of water molecule.

The temperature range between $-7{ }^{\circ} \mathrm{C} \sim 0{ }^{\circ} \mathrm{C}$ is generally called the "zone of maximum ice crystal formation". This means that within this temperature range, water-containing materials such as foods, cells, and tissues rapidly develop internal ice crystals ${ }^{[2]}$. Since rapid growth effectively destroys the inner structure of the materials, this temperature zone is not preferable for freezing storage. Conventionally, this problem was overcome using deep freezers that operate at $-60{ }^{\circ} \mathrm{C} \sim-80^{\circ} \mathrm{C}$ or liquid nitrogen $\left(\mathrm{LN}_{2},-196^{\circ} \mathrm{C}\right)$, as they reduce the exposure time of the materials to temperatures that promote ice crystal formation thereby minimizing crystal formation (Figure 1B). The minute ice crystals that form at these lower temperatures do not effectively destroy the internal structure and allow preservation of quality and/or activity of these materials in the deep freezer or $\mathrm{LN}_{2}$. Special refrigerators that mechanically inhibit ice crystal growth have also been developed.

Although these techniques are useful in sample preservation, they generate carbon dioxide $\left(\mathrm{CO}_{2}\right)$. Global reduction of $\mathrm{CO}_{2}$ is a priority in addressing global warming. In seeking an alternative cryopreservation strategy, we began considering

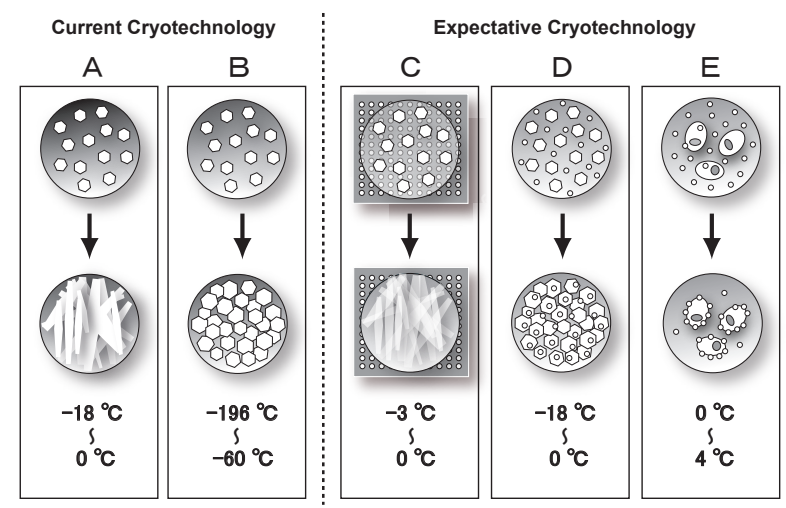

Fig. 1 Comparison between current and expected cryotechnologies.

Large circles in panels A D represent supercooled water, and small hexagons indicate ice nuclei. The small circles shown in panels $\mathrm{C} \sim \mathrm{E}$ represent AFP. Two large squares illustrated in $C$ represent icenucleation plate by assembly of AFP. Large circle in E represents cellpreservation fluid containing AFP. A: Infinite numbers of ice nucleus are naturally created in supercooled water between $-18{ }^{\circ} \mathrm{C}$ and $0{ }^{\circ} \mathrm{C}$ in a general freezing device. Each ice nucleus undergoes crystal growth, and the resultant polycrystalline occupies the whole space of water. B: Use of extremely low temperature $\left(-196^{\circ} \mathrm{C}\right.$ to $\left.-60^{\circ} \mathrm{C}\right)$ can effectively inhibit ice crystal growth, although it needs high-energy cost. C: AFPassembled plate effectively freezes the attached water near $0{ }^{\circ} \mathrm{C}$. D: AFP can inhibit the ice crystal growth strongly even in general freezing device. E: AFP increases the viability of various cells near $0{ }^{\circ} \mathrm{C}$.

Research Institute of Genome-based Biofactory, AIST Tsukisamu-Higashi 2-17-2-1, Toyohira-ku, Sapporo 062-8517, Japan * E-mail : s.tsuda@aist.go.jp. 
AFP. For example, an AFP-assembled plate (Figure 1C) may be capable of ice-nucleation, enabling efficient freezing of attached water near $0{ }^{\circ} \mathrm{C}$ (Figure 1A). Strong inhibition of ice growth by AFP in home freezer (Figure 1D) and cell preservation with AFP near $0{ }^{\circ} \mathrm{C}$ (Figure 1E) ${ }^{[3,4]}$ also suggests the potential for AFP as an environment-friendly cryotechnology.

Since large amounts of AFP are required to achieve this goal, we conducted studies to develop a mass preparation method for AFP. In addition, we explored the potential of AFP in sample cryopreservation for industry and medicine.

\section{Background}

Organisms produce a variety of proteins with various functions including metabolism, transportation, storage, structural formation, and immunoreaction. Many enzymes are utilized commercially and medically. Proteins recovered from extreme environments including volcanoes, hydrothermal vents, deep sea, deserts, polar regions, and certain hazardous materials have industrial potential. AFP is an example of such protein. AFP was discovered in 1969 in the serum of Antarctic cold-adaptive fish ${ }^{[5]}$. Similar ice crystallization inhibition is demonstrated with biosurfactants ${ }^{[6]}$ and poly vinyl alcohol ${ }^{[7]}$, but their property (determined as thermal hysteresis activity ${ }^{[3]}$ ) is markedly weaker than that of AFP.

Modern technological developments have dramatically improved instrument sensitivity, reducing the amount of sample needed for experimental determinations. For example, $1 \mu \mathrm{g}$ of protein is sufficient to analyze amino acid sequence and few milligrams enable determination of threedimensional (3D) structure by employing nuclear magnetic resonance and X-ray diffraction techniques. Biochemically, $10 \sim 20 \mathrm{mg}$ of protein represents a large amount ${ }^{[8]}$, and techniques that yield quantities in grams or kilograms were not considered a priority. However, such quantities are necessary to enable AFP research to advance its utility in different fields such as engineering, medicine, and food technology.

Biotechnology has long been concerned with large-scale purification of target proteins ${ }^{[9]}$. Genetic engineering combined with large-scale culturing of the transformed cells has achieved production of commercial quantities of several proteins such as cold temperature active enzymes used in laundry detergent, starch processing, pharmaceutical proteins, diagnostic antibodies, and bio-ethanol. However, large-scale preparation of genetically engineered proteins is difficult ${ }^{[9]}$. Recognizing these continuing challenges, we decided at present it would be most prudent to consider other methods such as chemical synthesis and purification to massproduce AFP from natural sources.

\section{Scenario}

Researchers from many different fields including biology, biochemistry, molecular biology, ice physics, biophysics, structural biology, and computer chemistry have studied AFP, and many papers have been published in reputable journals. Potential applicability of AFP in industrial and medical fields has been suggested ${ }^{[10]}$. However, this potential has not yet been realized. Production of AFP still involves using needle and syringe to collect the protein from serum of live fish. This arduous method and low product yield are reflected in the high purchase price of AFP (approximately USD 10/mg).

Typically, AFP is collected on-site (during Arctic fishing) and transported south. However, we have observed that fish near Japan $\left(\sim 43^{\circ} \mathrm{N}\right)$ also produce AFP ${ }^{[1]}$. To investigate this
A

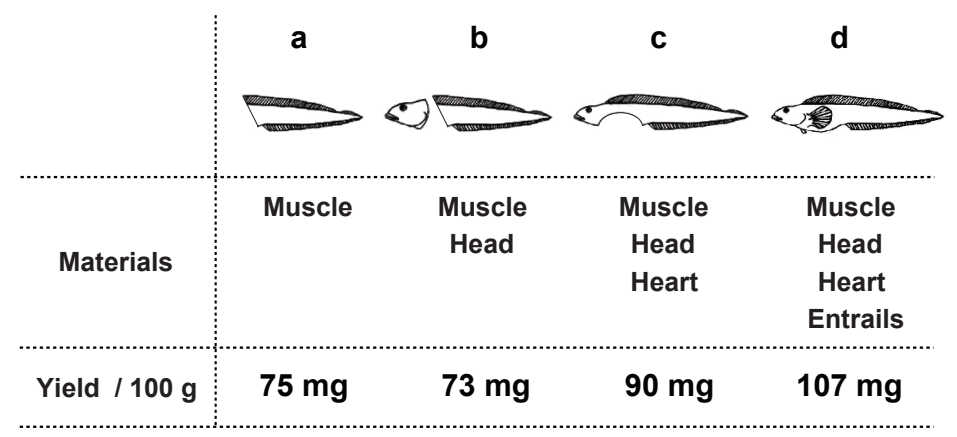

B

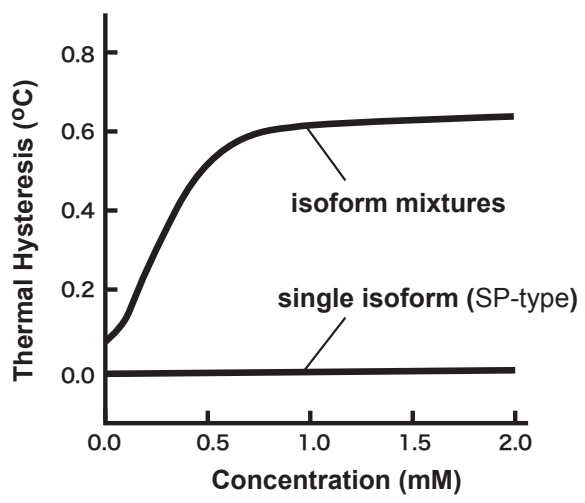

Fig. 2 Yield and antifreeze activity of AFP.

A: Results of total purified AFP (yield) per $100 \mathrm{~g}$ of different portions of an AFP-containing fish (material). B: Dependence of thermal hysteresis activity (i.e. antifreeze activity) on AFP concentration. A less active AFP isoform (SP-type) exhibits a substantial level of activity by addition of small amount of active AFP isoform ${ }^{[12]}$. 
further, we conducted an extensive survey of cold-adapted organisms in Japanese coastal waters with assistance of the Marine Bio-Institute of Sapporo Medical University and the Federation of Fisheries Cooperative Association of Notsuke. Various samples were also purchased from food stores and insect shops in Sapporo City. AFP activity was assayed using a photomicroscope system that enables detection of the ice-shaping ability using only $1 \mu \mathrm{L}$ of sample ${ }^{[1]}$. Over 160 species of Japanese fish were examined, of which at least 50 species contained AFP. In addition, AFP was found in tissues of Japanese plants (wheat), insects (stag beetles), and fungi (basidiomycete).

Interestingly, AFP activity was detected in fish fillets, minced fish, and dried fish purchased from food markets. On detailed examination, it was found that AFP could be purified from fish muscle as well as from serum (Figure 2A). The figure shows that larger amount of AFP was purified from samples obtained from heart tissue. Since presence of high amounts of contaminants such as lipids, enzymes, and sticky substances necessitated additional purification steps, which lowered the preparation efficiency of AFP, fish muscles may represent the best choice for mass preparation of AFP.

AFP isolated from the Japanese fish was compared with the established version recovered from cold-adapted polar species for DNA and amino acid sequences, 3D structure, and ice binding ability. Although these analyses are ongoing, so far it was found that the amino acid sequences were similar, allowing their grouping as Types I III. Similar result was also obtained for AFP from Japanese insects. Every Japanese organism expressed AFP as isoform mixture, as was the case for AFP from polar organisms. Especially, sample of eelpout (a species that lives off the east coast of Japan) was found to produce at least 13 isoforms of type III AFP. Initially, the presence of such large number of isoforms

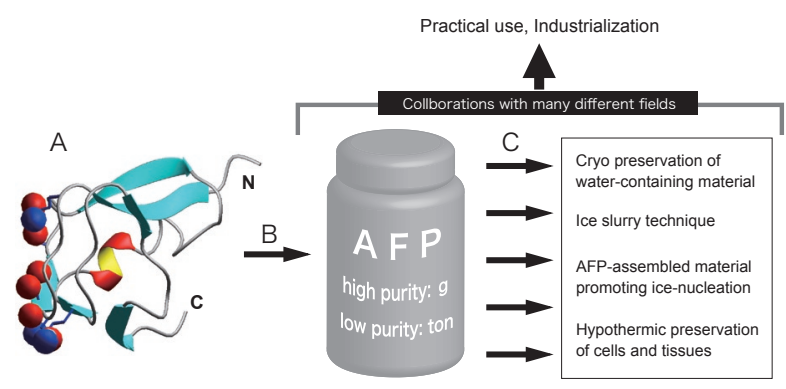

Fig. 3 Example of "Full Research" for AFP.

A: Biochemistry and molecular biology of AFP (Type 1 Basic Research). The ribbon representation shows a structure of type III AFP $($ PDB code $=1 \mathrm{MSI})$ where polar atoms colored in red and blue constitute an ice-binding surface. B: Development of mass preparation technique of AFP (Type 2 Basic Research). C: High yields of AFP allow research collaborations with researchers from different backgrounds, leading to practical use and industrialization of AFP. was perplexing. However, isoform mixtures of AFP possess higher antifreeze activity (thermal hysteresis) than single isoform ${ }^{[12]}$. Even an isoform with poor individual activity displayed enhanced activity in the presence of small amount of higher activity isoform (Figure 2B). Such cooperative enhancement was also identified for cell preservation activity of AFP in hypothermic conditions ${ }^{[13]}$. One should note that it is difficult to produce isoform mixture of AFP by genetic engineering and chemical synthesis.

The above findings prompted the scenario summarized in Figure 3 for practical use of AFP. The characterization and functional analysis of Japanese AFPs define the direction of the development of AFP technology (Figure 3A). Next, preparation of relatively large amounts of AFP isoform mixture can be achieved using fish muscles as raw material (Figure 3B). The isoform mixture can be used to perform various tests (Figure 3C), and holds potential for scale-up to industrial quantities.

\section{Factors that determine technology}

Highly purified sample is indispensable for research. In contrast, even poorly purified sample is generally sufficient for industrial and commercial use. In fact, enzyme used in detergents and food processing is partially purified product of cultivation ${ }^{[9]}$, offering an attractive saving in preparation costs. Partially purified AFP can also be utilized in food industry and in ice slurries, since AFP functions in a solution containing large amount of impurities. In the rules and regulations of the Japanese food industry, partially purified AFP is categorized as natural extract while highly purified AFP is considered food additive. The former can be applied directly to various foods after careful safety check

\begin{tabular}{|c|c|c|c|c|c}
\hline & $\mathrm{A}$ & $\mathrm{B}$ & $\mathrm{C}$ & $\mathrm{D}$ & $\cdots$ \\
\hline Activity & ++ & + & - & ++ & \\
\hline Resource availability & -- & ++ & - & + & \\
\hline Molecular size & - & + & ++ & - & \\
\hline Structural rigidity & ++ & -- & ++ & ++ & \\
\hline Infrastructure & -- & ++ & -- & + & \\
\hline Acid/alkaline tolerance & ++ & - & - & ++ & \\
\hline Heat Stability & ++ & ++ & ++ & -- & \\
\hline$\vdots$ & & & & &
\end{tabular}

Fig. 4 Evaluations of factors such as activity and resource availability for AFP species (isoform mixture) from fish A, B, C, and so on, needed for technological developments.

We divided the evaluation results into four grades: ++ (excellent), + (good), - (poor), and -- (very poor) (see text). The evaluation for element indicated by thick-framed box is an ordinary biochemical study. Activity and resource availability are found to be especially important for advancing the technological developments of AFP. 
based on experience and knowledge of the manufacturing company, while the latter requires stringent tests before approval. This implies that highly purified AFP cannot be used for all technologies utilizing AFP. On the other hand, highly purified AFP is indispensable for preparation of cell preservation fluid or AFP-assembled ice nucleation plate (see below). Any initial examination of AFP, even in food technology, requires highly purified AFP to obtain detailed information of its effects. Therefore, in this study, we focused on the technological developments of both crude and highly purified AFP. Commercial use of the crude form would require production capacities in tons.

Technological developments are in progress according to the information shown in Figure 4. The columns show the species of fish (source materials) that contain AFP as mixture of isoforms, and the rows (such as activity and resource availability) are the factors needed for technological developments. The initial step of development is to deduce the activity of AFP sample from "A" (indicated by thickframed box). In this step, sequential determinations and activity assay are done by preparing minute amount of sample, which provides evaluations for rows in column A (excellent $[++]$, good $[+]$, poor $[-]$, and very poor [--]) with regard to technological developments. Further examinations for molecular size, 3D structure, acid/alkaline tolerance, heat stability, as three examples, are undertaken. Smaller molecular size, rigid structure, high acid/alkaline tolerance, and high heat stability are preferred in AFP technologies. This approach is similar to the one used in biochemical evaluations of sample. A noticeable point is that Figure 4 contains information that are irrelevant to academic research such as evaluations regarding the availability of natural resources and infrastructure (e.g. fishermen's union, fish sausage factory, storage warehouse, and marketing channel). However, all such information are important determinants for technological developments. In case of Figure 4, AFP from A will not become a target of development since its resource availability is very poor even though it has excellent activity. In contrast, AFP from B becomes the target although its activity is inferior to AFP-A, since sufficient resource availability compensates for the inferiority. For AFP-B, mass preparation method as well as AFP technology can be developed by utilizing its high thermal stability. AFP-C and AFP-D can also be developed since their resource availabilities are adequate. For AFP-C, poor infrastructure availability may raise the product cost. Although the significance for development of various factors differs, it may be concluded that availability of source material as well as activity are the principle determinants of technological development.

We tried to update Figure 4 based on the increasing knowledge of AFP, which is reflected in the increasing number of columns with time. The factors in the row will also be appended with time concerning safety (toxicity), shelf life, and recyclability of AFP as three examples.

\section{Results}

Figure 5 summarizes the elements relevant to mass preparation of AFP, such as AFP species (source materials),

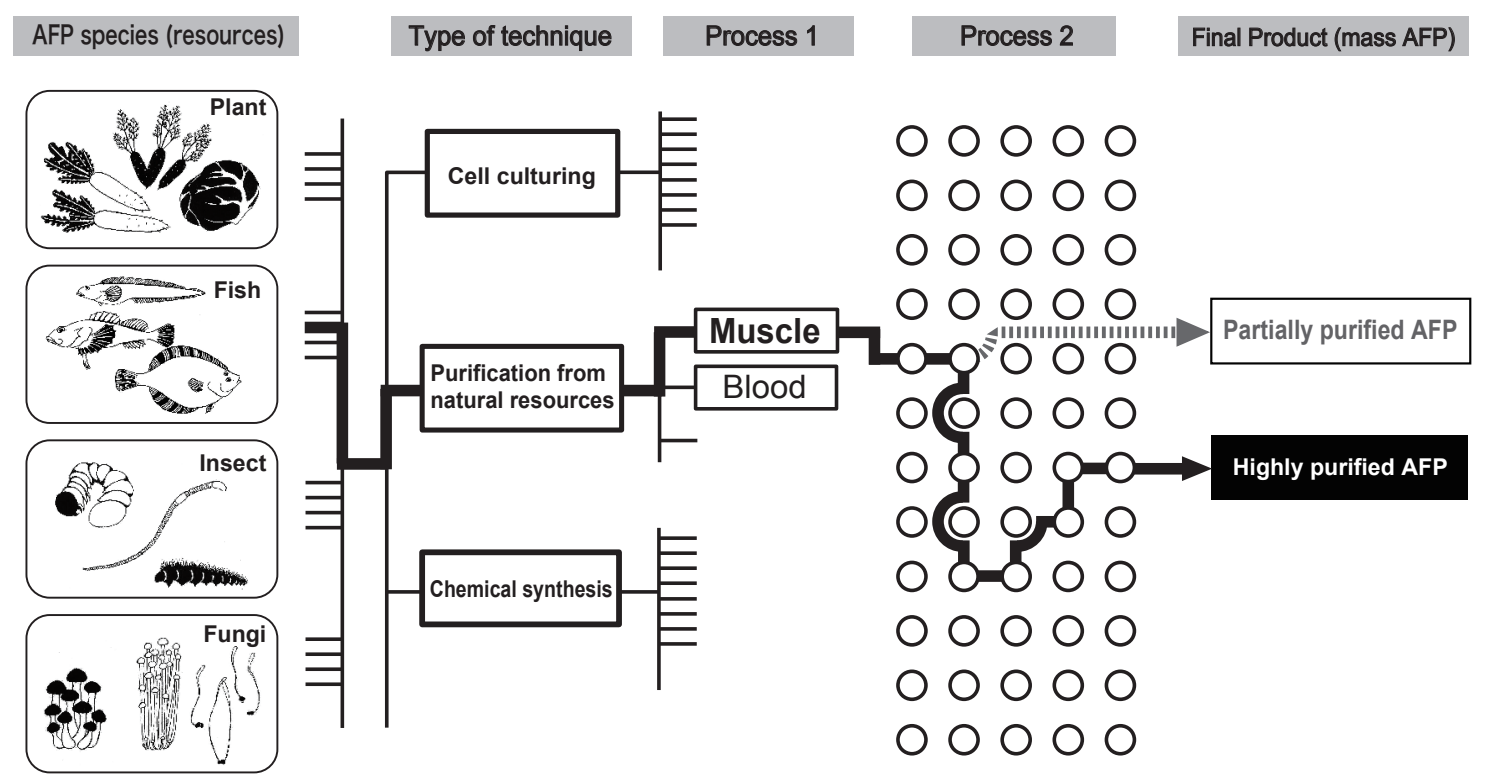

Fig. 5 Procedure for obtaining mass amount of AFP (thick arrows), which can also be used for collaborative studies and industrialization.

Open circles ( $\bigcirc$ ) shown in Process 2 indicate detailed methods to purity AFP such as heat denaturation, centrifugation, filtering, and chromatography. Initial methods in Process 2 are common for both partially and highly purified samples of AFP. 
technique, and purification processes. The arrows connect our present choices of elements, thereby indicating procedures to obtain highly purified AFP (thick arrow) and partially purified AFP (broken arrow). The open circles (O) indicate detailed methods to purity AFP such as heat denaturation, centrifugation, filtering, and chromatography, which were carried out using suspension fluid of minced muscle of AFP-containing species of fish. These methods were initially chosen based on Figure 4 and were further optimized by considering cost and time required to improve the preparation efficiency. Removal of certain procedures and rearranging others also contributed to improvement. For example, high-performance liquid chromatography (HPLC) was not selected because of its inefficiency and high operational costs.

As described, we chose AFP-containing fish as source material. After considering the quantity of fish resources and their price, we selected three species of edible fish which were captured at a fishery located on the east coast of Hokkaido and for which involvement of AFP types I III were identified in our laboratory. These fish were not captured as merchandise but were "by-catches" of scallops and shrimps (i.e. they were food for by-catch fish). The by-catch fish are typically discarded as they have no market value. We were able to secure at least three tons of by-catch fish during the winter. Minced muscle was transferred to a cold storage warehouse for storage, and samples were transported to the laboratory as needed. For type III AFP, our efficiency of purification was approximately $3 \mathrm{~g}$ (99\% purity)/5 days/1 person. The collaborating company prepared crude AFP samples at 200-times higher efficiency than our laboratory, but this was still not the achievable upper limit. Figure 6A
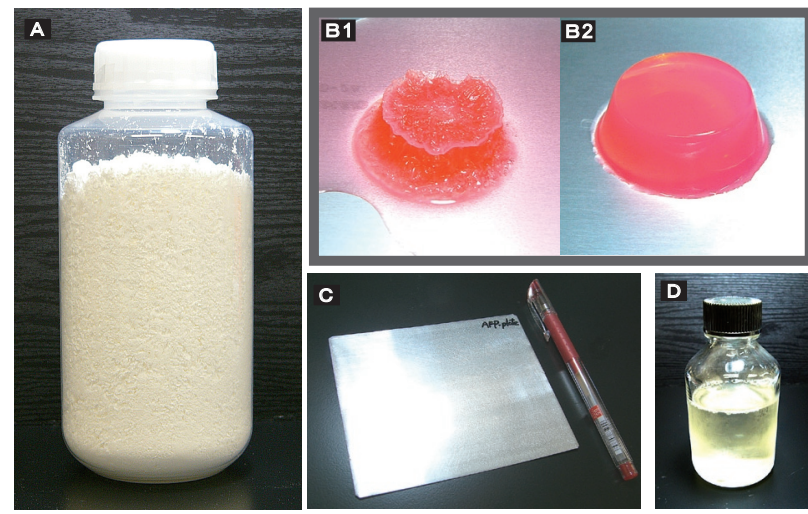

Fig. 6 Outcomes of the present study.

A: Approximately $11 \mathrm{~g}$ of highly purified type III AFP. The preparation efficiency of this sample is $3 \mathrm{~g} / 5$ days/person. B: Photographs of agarose gel in absence (B1) and presence of AFP (B2) after cold storage $\left(-18^{\circ} \mathrm{C}\right)$. Addition of AFP can provide freeze tolerance to many water-containing materials. C: AFP-assembled aluminum plate that exerts ice-nucleation function. D: Cell preservation fluid containing AFP, which dramatically improves cell viability under hypothermic condition $\left(0^{\circ} \mathrm{C}\right)$. shows the appearance of approximately $10 \mathrm{~g}$ of high purity sample of type III AFP. Currently, we have amassed $240 \mathrm{~g}$ with market value of approximately USD 3 million. Raw material and preparation costs were extremely low.

Acquisition of gram quantities of AFP enabled us to examine the cryopreservation effect of AFP on various watercontaining substances such as processed food, soups, icesweets, noodles, bread, soft drinks, alcohol beverages, drugs, cosmetics, inks, polymer gels, polymer membranes, vegetables, fruits, seeds, meats, and seafood. Although the examinations are not complete, in principle, they are expected to become freeze tolerant by addition of AFP. For substance such as meat whose inner structure is complex, it is difficult to transfer AFP internally. Once the meat is minced, however, distribution of AFP is facilitated, and freeze tolerance can be expected.

Figure 6 shows some examples. Figure 6B1 is a photograph of agarose gel after overnight storage at $-18{ }^{\circ} \mathrm{C}$ in a home freezer. As frozen gel began to thaw, water flowed out from the inside of the gel. This occurred because the agarose network of gel was destroyed by growth of ice crystals (Figure 1A). Similar phenomenon was observed in the thawing of frozen meats, fruits, and vegetables, and this water flow is called drip in meats. We found that addition of slight amount of AFP effectively preserved the inner structure of frozen gel and stopped the water flow, as shown in B2. This was attributed to the inhibition of ice crystal growth by strong binding of AFP to crystal surface (Figure 1D). It should be noted that strong preservation effect of AFP was observed in the temperature zone of maximum ice crystal formation, between $-7{ }^{\circ} \mathrm{C}$ and $0{ }^{\circ} \mathrm{C}$, implying that AFP can replace $\mathrm{LN}_{2}$ for high quality preservation of water-containing materials, and this may further save energy and reduce $\mathrm{CO}_{2}$ emissions.

In this study, type III AFP was chemically or physically immobilized on a surface plane of metal by spraying a solution of highly purified protein (Figure 3). This type of AFP can form an ice-binding surface (represented by red and blue CPKs in Figure 3A), which binds specifically to a set of oxygen atoms of ice crystals ${ }^{[14]}$. This ice-binding site is located on the opposite side of the N-terminal end of this molecule. Given this knowledge, we assumed that if we connected the N-terminal end of type III AFP to the metal surface, the ice-binding surface would be directed outside of the metal surface. When performing this experiment, numerous AFP molecules should, in practice, be connected to the metal surface in that manner, creating a fairly large ice-binding surface on metal due to assembly of type III AFP molecules. Our assumption is that such large ice-binding surface will cause the assembly of ice-like structure in water placed on the surface. In other words, the AFP-assembled surface will have ice-nucleation function. Figure $6 \mathrm{C}$ is a photograph of an aluminum plate on which approximately 
$6.0 \times 10^{11}$ type III AFPs were assembled per square $\mathrm{cm}$. Detailed descriptions of this experiment will be presented in a separate paper. Here, we show some of the results. First, a droplet of water placed on the AFP-assembled plate froze at approximately $5{ }^{\circ} \mathrm{C}$ higher temperature than the unassembled plate, consistent with ice-nucleation ability of the surface. Second, unidirectional freezing occurred from the surface of AFP-assembled plate, which led to formation of extremely clear ice. AFP could be assembled not only on flat metal surface but also on curved substances and particles in various sizes.

Figure 6D is a photograph of cell preservation fluid containing AFP. Human and animal cells could not maintain their functions in vitro for a prolonged time. In the fields of organ transplant and regenerative medicine, tremendous efforts are spent to achieve long-term preservation of cells and organs in both frozen and unfrozen states. Earlier in this report, we explained the effectiveness of quick-freezing using very low temperature (e.g. $\mathrm{LN}_{2}$ ) for preservation of frozen substance (Figure 1). Here, we briefly report our results of cell preservation in unfrozen state, which will be useful in 1 21 day period before transplantation of cultured cells. We attempted to preserve approximately 10,000 unfrozen $\left(0{ }^{\circ} \mathrm{C}\right)$ human hepatoma cells (HepG2) without AFP. Ninety percent of the HepG2 cells died within $12 \mathrm{~h}$ using commercially available preservation fluid. In contrast, AFP-containing preservation fluid (Figure 6D) preserved $90 \%$ of the HepG2 cells even after $72 \mathrm{~h}^{[13]}$. This preservative effect of AFP was also identified in cell lines of small intestine, kidney, umbilical cord, blood, cervix, and pleural effusion. Gram quantities of highly purified AFP were sufficient to examine the preservation effect on cells, but were insufficient for tissue and organ examinations. Further study is necessary to overcome this problem.

\section{Future development}

Amount of protein that may produce superior performance at molecular level can be insufficient for practical use. In other words, quantity has been a hindrance for expansion of basic research into practical technology. In the case of AFP, the ability to generate grams of product enables collaborative advances in different fields such as food, medicine, and engineering. More collaborations are expected, and product utilization of AFP is becoming a reality. We note with interest that the currently reported technique that brings the benefits of lowered energy consumption and $\mathrm{CO}_{2}$ emission is derived from the classical extraction of target protein from natural resources. At the same time, our technique utilizes advanced studies from molecular biology to 3D structural analysis.

Applications of AFP in medical fields will require approval from the appropriate government agencies concerning aspects that include toxicity, mutagenicity, and carcinogenicity. These approvals may require time. It will also be necessary to construct AFP preparation facility that satisfies the regulation of Good Manufacturing Practice (GMP). Although much remains to be done, this study is an encouraging start.

In addition to applications in food industry, partially purified AFP may be used in the cold storage systems in office buildings. Most air conditioners work by lowering the temperature of a building through circulation of refrigerant. Replacement of refrigerant with ice slurry would save energy while maintaining the same level of air conditioning. Partially purified AFPs would prevent aggregation of ice slurry that often occurs during circulation.

Many biological compounds including antifreeze proteins have potential industrial applications. However, sufficient quantities of these compounds are required for research to further investigate their applications. AFP is one example. Bio-ethanol is another. This study was primarily based on the functional analysis of protein from organisms of Hokkaido. In July 2008, the G8 Summit was held at Lake Toya in Hokkaido. We would like to contribute to reducing global warming through our technologies.

\section{Acknowledgements}

We thank Dr. Takaaki Inada (Energy Technology Research Institute (ETRI), AIST), Dr. Shuichiro Matsumoto, Prof. Michiaki Matsushita, and Prof. Satoru Todo (General and Digestive Surgery, Graduate School of Medicine, Hokkaido University) for many helpful advices and discussions. We also thank Fumie Shiraishi, Etsuko Hayashi, and Michiko Ito for developments of mass preparation techniques of AFP.

\section{References}

[1] P.V. Hobbs: Ice Physics, 18-39, Oxford University Press, London (1974).

[2] H.Tsuyuki: Shokuhin kakogaku dai 2 han-kako kara hozo made -(Processing of Food: From Processing to Storage), 14-16, Kyoritsu Press, Tokyo (1990) (in Japanese).

[3] Y. Yeh, and R.E. Feeney: Antifreeze proteins: Structures and mechanisms of function, Chemical Reviews, 92(2), 601-617 (1996).

[4] B. Rubinsky, A. Arav, and G.L. Fletcher: Hypothermic protection: A fundamental property of "antifreeze" proteins, Biochem. Biophys. Res. Commun., 180(2), 566-571 (1991).

[5] Z. Jia, and P.L. Davies: Antifreeze proteins: an unusual receptor-ligand interaction, Trends Biochem. Sci., 27, 101-106 (2002).

[6] D. Kitamoto, H. Yanagishita, A. Endo, M. Nakaiwa, T. Nakane, and T. Akiya: Remarkable antiagglomeration effect of a yeast biosurfactant, diacylmannosylerythritol, on icewater slurry for cold thermal storage, Biotechnol. Prog., 17, 362-365 (2001). 
[7] T. Inada and S.-S. Lu: Thermal hysteresis caused by nonequilibrium antifreeze activity of poly(vinyl alcohol), Chem. Phys. Lett., 394, 361-365 (2004).

[8] M. Okada, K. Miyazaki, eds: Kaitei dai3pan tanpakushitsu jikken noto (jokan)-chushitsu. bunri to kumikae tanpakushitsu no hatsugen-(Protein Experiment Note, 3rd Edition: Extraction, Separation, and Gene Expression of a Protein), 202, Yodo Press (2004) (in Japanese).

[9] M. Nomoto:Koso kogaku (The Enzyme Technology), Gakkai Press Center, Tokyo (1993) (in Japanese).

[10] R.E. Feeney and Y. Yeh: Antifreeze proteins: Current status and possible food uses, Trends Food Sci Tech., 9, 102-106 (1998).

[11] M. Takamichi, Y. Nishimiya, A. Miura and S. Tsuda: Effect of annealing time of an ice crystal on the activity of type III antifreeze protein, FEBS J., 274 (24), 6469-6476 (2007).

[12] Y. Nishimiya, R. Sato, M. Takamichi, A. Miura and S. Tsuda: Co-operative effect of the isoforms of type III antifreeze protein expressed in notched-fin eelpout, Zoarces elongatus Kner, FEBS J., 272, 482-492 (2005).

[13] Y. Hirano, Y. Nishimiya, S. Matsumoto, M. Matsushita, S. Todo, A. Miura, Y. Komatsu and S. Tsuda: Hypothermic preservation effect on mammalian cells of type III antifreeze protein from notched-fin eelpout, Cryobiology (in press).

[14] Y. Nishimiya, S. Ohgiya and S. Tsuda: Artificial multimers of the type III antifreeze protein: effects on thermal hysteresis and ice crystal morphology, J. Biol. Chem., 278 (34), 32307-32312 (2003).

[15] Y. Mie, Y. Nishimiya, F. Mizutani and S. Tsuda: Assembly of antifreeze protein reveals the ice nucleation activity, (in preparation).

Received original manuscript September 18,2007 Revisions received November 19,2007 Accepted November 19,2007

\section{Authors}

\section{Yoshiyuki Nishimiya}

Functional Protein Research Group, Research Institute of Genome-based Biofactory of AIST

Senior researcher (2000-present). Research interests are identification, functional analysis, mass-preparation method, and practical application of antifreeze protein. Main research fields are molecular biology and directed molecular evolution. Ph.D. Graduate School of Engineering, Tohoku University (2000).

\section{Yasuhiro Mie}

Biomolecular Engineering Group, Research Institute of Genome-based Biofactory of AIST

Researcher (2005-present). Research interests are assembly of proteins on a surface that functions as useful material and tool. Electron transfer between electrode and metalloprotein. Main research fields are electrochemistry and biomedical engineering (biosensor). Ph.D. Graduate School of Engineering, Kumamoto University (2000).

\section{Yu Hirano}

Biomolecular Engineering Group, Research Institute of Genome-based Biofactory of AIST

Researcher (2005-present). Research interests are development of scanning electrochemical microscopy for the analysis of cell preservation mechanism by antifreeze protein. Main research fields are electrochemistry and biomedical engineering (biosensor). Education: Ph.D. Graduate School of Engineering, Tohoku University (2005).

\section{Hidemasa Kondo}

Functional Protein Research Group, Research Institute of Genome-based Biofactory of AIST

Senior researcher (2005-present). Research interests are elucidation of molecular mechanism of antifreeze protein and industrial enzyme by using X-ray crystallography. Main research fields are protein crystallography and structural biology. Ph.D. Graduate School of Science, Hokkaido University (1997).

\section{Ai Miura}

Functional Protein Research Group, Research Institute of Genome-based Biofactory of AIST

Research Assistant (1996-present). Research interests are new exploration of fish antifreeze protein and its technological applications (frozen foods, ice slurry, etc.). Main research fields are preparation and analysis of two- and three-dimensional NMR spectra of a protein. Dietitian (1994).

\section{Sakae Tsuda}

Functional Protein Research Group, Research Institute of Genome-based Biofactory of AIST

Group leader (2001-present) and professor (2003-present). Research interests are low-temperature biology, protein-ice interaction, and development of energy-saving technologies. Main research fields are multidimensional NMR spectroscopy and structural biology. Ph.D. Graduate School of Science, Hokkaido University (1993).

\section{Discussion with reviewers}

\section{Quantitative Aspects}

\section{Question (Koichi Mizuno)}

With regard to the ice-nucleation ability of AFP-assembled aluminum plate, is it possible to numerically quantify the superiority of this plate compared to other materials for freezing water near $0{ }^{\circ} \mathrm{C}$ ?

\section{Answer (Sakae Tsuda)}

It is very difficult for us to give concrete answer to your question. Suppose that our plate can freeze a $1 \mathrm{~mL}$ water droplet at $-1{ }^{\circ} \mathrm{C}$, for which $-18{ }^{\circ} \mathrm{C}$ of $\mathrm{Tf}$ was evaluated when the plate was not used, we need to evaluate the amount of energetic barrier which causes ice-nucleation in a water droplet. This energetic barrier, however, depends on many parameters, such as cooling rate, environmental perturbations, and contaminants, which can hardly be evaluated. As for difference in potential energy, approximately $40 \mathrm{~J}$ is estimated for $1 \mathrm{~mL}$ of ice crystal between -18 and $-1{ }^{\circ} \mathrm{C}$. Please note that we avoided complete descriptions of the performance of our developments due to future patentability.

\section{Cell preservation ability}

Question (Koichi Mizuno)

Did the authors attempt preservation of hepatocells (HepG2) near $0{ }^{\circ} \mathrm{C}$ without freezing them? The fact that $90 \%$ of HepG2 cells die in 12 hours using commercial fluid seemed to me that 0 ${ }^{\circ} \mathrm{C}$ is not suitable for cell preservation. I imagine that AFP may be having some biological influence on the cells. Has detailed mechanism of such influence been clarified?

\section{Answer (Sakae Tsuda)}

The method to preserve cells and tissues from heart, liver, and kidney for $1 \sim 24$ hours near $0{ }^{\circ} \mathrm{C}$ without freezing has actually been utilized in the medical fields, such as transplantation 
and regeneration. This is called "hypothermic preservation". It is important to have a certain period (1 10 days) before transplanting the cells to the patient, because the cells must reach culturing limit. The cell preservation activity of AFP is not common knowledge so far, since many papers report that AFP sometimes exerts no expectative activity, or performs worse than other substances. We think such uncertainties are due to insufficient amount of AFP needed to verify the reproducibility of data, and also due to imperfections in conducting this type of delicate experiments. Although some models have been proposed, it is necessary to clarify the cell preservation mechanism of AFP, and our AFP preparations in certain quantities are expected to contribute to future studies. 